\title{
CORONARY ARTERIAL SIZE LATE AFTER THE ATRIAL INVERSION PROCEDURE FOR TRANSPOSITION OF THE GREAT ARTERIES: IMPLICATIONS FOR THE ARTERIAL SWITCH OPERATION
}

Zahid Amin, MD

Doff B. McElhinney, $\mathrm{MD}^{\mathrm{c}}$

Philip Moore, $\mathrm{MD}^{\mathrm{b}}$

V. Mohan Reddy, $\mathrm{MD}^{\mathrm{c}}$

Frank L. Hanley, MD
Background: Coronary flow reserve in the hypertrophied ventricle is reduced. One contributing factor may be the size of the proximal coronary arteries. In patients who undergo atrial inversion procedures for transposition of the great arteries, the left coronary artery supplies the pulmonary ventricle and may be smaller than the right coronary artery. We hypothesized that the dimensions of the coronary arteries may correlate with symptomatic status after atrial inversion and may be an important factor when these patients are considered for the arterial switch operation.

Methods: The proximal left and right coronary arteries were measured in 9 patients with transposition and failure of the systemic right ventricle after atrial inversion, 10 asymptomatic patients after atrial inversion, and 10 patients with normal hearts. The diameters of the coronary arteries were indexed to body surface area and compared.

Results: The absolute and indexed diameters of the right coronary artery were greater in symptomatic patients than in asymptomatic patients (indexed: $3.1 \pm 0.6$ vs $\left.2.4 \pm 0.4 \mathrm{~mm} / \mathrm{m}^{2}, P<.001\right)$ or control patients $(2.0 \pm$ $0.3, P<.001)$, and the absolute diameter of the left coronary artery was smaller $(2.9 \pm 0.7$ vs $3.6 \pm 0.5 \mathrm{~mm}, P=.003$ [asymptomatic], $3.6 \pm 0.5 \mathrm{~mm}$, $P=.01$ [control]). In symptomatic patients, the absolute and indexed diameters of the left coronary artery were smaller than those of the right (indexed: $2.1 \pm 0.6$ vs $\left.3.1 \pm 0.6 \mathrm{~mm} / \mathrm{m}^{2}, P<.001\right)$. By contrast, there was no difference in asymptomatic patients $\left(2.2 \pm 0.5\right.$ vs $\left.2.4 \pm 0.4 \mathrm{~mm} / \mathrm{m}^{2}, P=.44\right)$, and the left coronary artery was larger in normal control patients ( $2.2 \pm 0.4$ vs 2.0 $\left.\pm 0.3 \mathrm{~mm} / \mathrm{m}^{2}, P<.001\right)$.

Conclusions: Differences in the sizes of the proximal coronary arteries may be related to symptomatic status in patients with transposition of the great arteries who have undergone an atrial inversion procedure, as well as to the efficacy of ventricular retraining. When pulmonary artery banding and subsequent arterial switch are considered for patients with a Mustard or Senning procedure and a failing systemic right ventricle, the size of the proximal coronary arteries may be an important factor and should be evaluated with preoperative imaging studies. (J Thorac Cardiovasc Surg 2000;120:1047-52)
From the Division of Pediatric Cardiology, ${ }^{a}$ Medical College of Georgia, Augusta, Ga, and the Departments of Pediatrics ${ }^{\mathrm{b}}$ and Cardiothoracic Surgery, ${ }^{\mathrm{c}}$ University of California, San Francisco, Calif.

Read at the Eightieth Annual Meeting of The American Association for Thoracic Surgery, Toronto, Ontario, Canada, April 30-May 3, 2000.

Received for publication May 4, 2000; revisions requested July 24, 2000; revisions received Aug 18, 2000; accepted for publication Aug 25, 2000.

Address for reprints: Zahid Amin, MD, University of Nebraska/Creighton University, Children's Hospital, Joint Division of Pediatric Cardiology, 8301 Dodge St, Omaha, NE 68114 (E-mail: zamin@chsomaha.org).
The arterial switch operation is the standard procedure for the repair of transposition of great arteries (TGA) in the current era. ${ }^{1}$ Before the development of this procedure, rerouting of venous return by atrial inversion was the method of choice. In patients with a Mustard or Senning procedure, the right ventricle

Copyright $(2000$ by The American Association for Thoracic Surgery $0022-5223 / 2000 \$ 12.00+0 \quad \mathbf{1 2 / 6 / 1 1 1 1 7 4}$

doi: $10.1067 / \mathrm{mtc} .2000 .111174$ 
Table I. Comparison of demographic variables and absolute coronary artery diameters

\begin{tabular}{lccc}
\hline Variable & Symptomatic TGA & Asymptomatic TGA & Normal controls \\
\hline Age $(\mathrm{y})$ & $20.3 \pm 11.7$ & $15.9 \pm 2.2$ & $13.7 \pm 4.4$ \\
Body surface area $\left(\mathrm{m}^{2}\right)$ & $1.4 \pm 0.3$ & $1.6 \pm 0.2$ & $1.6 \pm 0.3$ \\
RCA diameter $(\mathrm{mm})$ & $4.3 \pm 0.8^{+}$ & $3.8 \pm 0.7$ & $3.2 \pm 0.3^{*}+\ddagger$ \\
LCA diameter $(\mathrm{mm})$ & $2.9 \pm 0.7$ & $3.6 \pm 0.5^{*}$ & $3.5 \pm 0.3^{*}$ \\
\hline
\end{tabular}

$R C A$, Right coronary artery; $L C A$, left coronary artery. All significant $(P<.05)$ statistical differences by general factorial analysis of variance are indicated:

$* P \leq .01$ versus symptomatic TGA.

$\dagger P \leq .01$ versus asymptomatic TGA.

$\ddagger P<.001$ versus LCA (same group).

pumps to the systemic circulation, although it may not be well suited as a systemic pump because of several morphologic features. As long-term follow-up has become available in patients who previously underwent repair of TGA with a Mustard or Senning procedure, it has become clear that a substantial proportion of them have supraventricular arrhythmias, systemic atrioventricular valve regurgitation, and failure of the systemic right ventricle. ${ }^{2}$ One management strategy that has been used in patients in whom systemic right ventricular failure develops after an atrial switch operation is left ventricular retraining by pulmonary artery banding, with a subsequent arterial switch operation. ${ }^{3-6}$

The sizes of the coronary arteries are linearly related to the size and thickness of the ventricles. ${ }^{7}$ Accordingly, in hearts with concordant ventriculoarterial connections, the left ventricle is thicker and has more mass than the right ventricle; by contrast, in patients with TGA who have undergone an atrial inversion procedure, the right ventricle is thicker than the left. ${ }^{8,9}$ Several prior studies have demonstrated differences in coronary arterial diameter in patients with TGA in comparison with normal control subjects. ${ }^{9,10}$ We hypothesized that the sizes of the coronary arteries may correlate with symptomatic status after atrial inversion and may be an important factor in the success of ventricular retraining and arterial switch in such patients.

\section{Methods}

Patients. Three groups of patients were studied: those with symptomatic TGA $(\mathrm{n}=9)$, those with asymptomatic TGA $(\mathrm{n}=10)$, and normal control subjects $(\mathrm{n}=10)$. Defining characteristics of each group are as follows: All patients with symptomatic TGA had undergone an atrial inversion procedure for TGA and had been referred for ventricular retraining and an arterial switch operation to the University of California, San Francisco, from 1993 to 1998. These patients had moderate to severe dysfunction of the systemic right ventricle and moderate or severe tricuspid regurgitation. They were in New York Heart Association (NYHA) class III or IV. Strict quantitative physiologic criteria for inclusion in this group were not applied.

All patients with asymptomatic TGA had undergone an atrial inversion procedure, had normal function or only trivial dysfunction of the systemic right ventricle, and had no more than trivial tricuspid regurgitation. These patients were in NYHA class I or II. They underwent cardiac catheterization for hemodynamic evaluation from 1993 to 1998.

All control patients had normal segmental cardiac anatomy with normal ventricular function and underwent cardiac catheterization either for suspected anomalous coronary artery or for evaluation of the coronary arteries several years after being treated for Kawasaki disease, from 1993 to 1998. In all of the control patients, the coronary arteries were found to be normal.

A right-dominant coronary arterial system was present in all 3 groups, without any evidence of stenosis. Mean age and body surface area did not differ between patients in the 3 groups (Table I).

Angiographic measurement of the coronary arteries. The diameters of the proximal right and left coronary arteries were measured off-line from selective coronary arterial or aortic root angiograms by an experienced pediatric cardiologist who was unaware of patient grouping. Each coronary artery was measured twice, $5 \mathrm{~mm}$ from its aortic origin, with the average of the 2 measurements recorded for analysis.

Data analysis. Diameters of the right and left coronary arteries were indexed to body surface area. The ratio of indexed right to indexed left coronary artery diameters was then calculated. Demographic variables, the absolute and indexed coronary artery diameters, and right to left coronary artery diameter ratios were compared among the 3 groups with the use of general factorial analysis of variance. Absolute and indexed left and right coronary artery diameters were compared within each group by means of paired $t$ test analysis. Data are presented as mean \pm standard deviation.

\section{Results}

Right coronary artery. The absolute diameter of the right coronary artery was significantly larger in patients with symptomatic and asymptomatic TGA than in controls (Table I). There was no difference in the absolute diameter of the right coronary artery between patients with symptomatic and asymptomatic TGA.

The diameter of the right coronary artery indexed to body surface area (right coronary artery index) was significantly larger in patients with symptomatic TGA than in either patients with asymptomatic TGA or in control patients (Fig 1, A). It was also larger in patients with 
asymptomatic TGA than in control patients (Fig 1, A).

Left coronary artery. The absolute diameter of the left coronary artery was significantly smaller in patients with symptomatic TGA than in either patients with asymptomatic TGA or in control patients, between whom there was no significant difference (Table I).

The diameter of the left coronary artery indexed to body surface area (left coronary artery index) did not differ significantly between any of the groups (Fig 1, B).

Relative size of the right and left coronary arteries. In patients with symptomatic TGA, the absolute and indexed diameters of the right coronary artery were significantly larger than those of the left coronary artery (Table I, Fig 2). In patients with asymptomatic TGA, there was no significant difference between the absolute diameters of the right and left coronary arteries or between the right and left coronary artery indices (Table I, Fig 2). In control patients, both the absolute and indexed diameters of the right coronary artery were significantly smaller than the absolute and indexed diameters of the left coronary artery (Table I, Fig 2).

The ratio of right coronary artery index to left coronary artery index was significantly larger in patients with symptomatic TGA than in either patients with asymptomatic TGA or in control patients (Fig 1,C). The right-toleft coronary artery index was larger in patients with asymptomatic TGA than in control patients to a degree that approached significance (Fig 1,C).

\section{Discussion}

For patients undergoing primary arterial switch, 10year survival is greater than $90 \% .^{1}$ In contrast, early and late mortality in patients who undergo ventricular retraining and arterial switch after an atrial inversion procedure is relatively high., ${ }^{3,4,6}$ Mavroudis and Backer $^{3}$ recently published a series of 11 patients who underwent pulmonary artery banding in preparation for conversion to an anatomic repair. Six of these patients were reported to undergo an arterial switch operation, with 2 deaths. Biventricular failure developed in 4 of the remaining patients, and they later received a heart transplant. Similarly, in the series reported by Prieto and associates, ${ }^{6}$ there was a $20 \%$ early mortality and a $20 \%$ late mortality associated with conversion of a Mustard or Senning procedure to an arterial switch. In addition, there seems to be a high dropout rate after pulmonary artery banding, with patients being listed for cardiac transplantation. , $^{3,4}$

Several factors may be responsible for the poor outcome in some patients in whom ventricular retraining and arterial switch is attempted. Conversion to an arterial switch is typically planned in these patients when they are in NYHA class III or IV because of systemic ven-
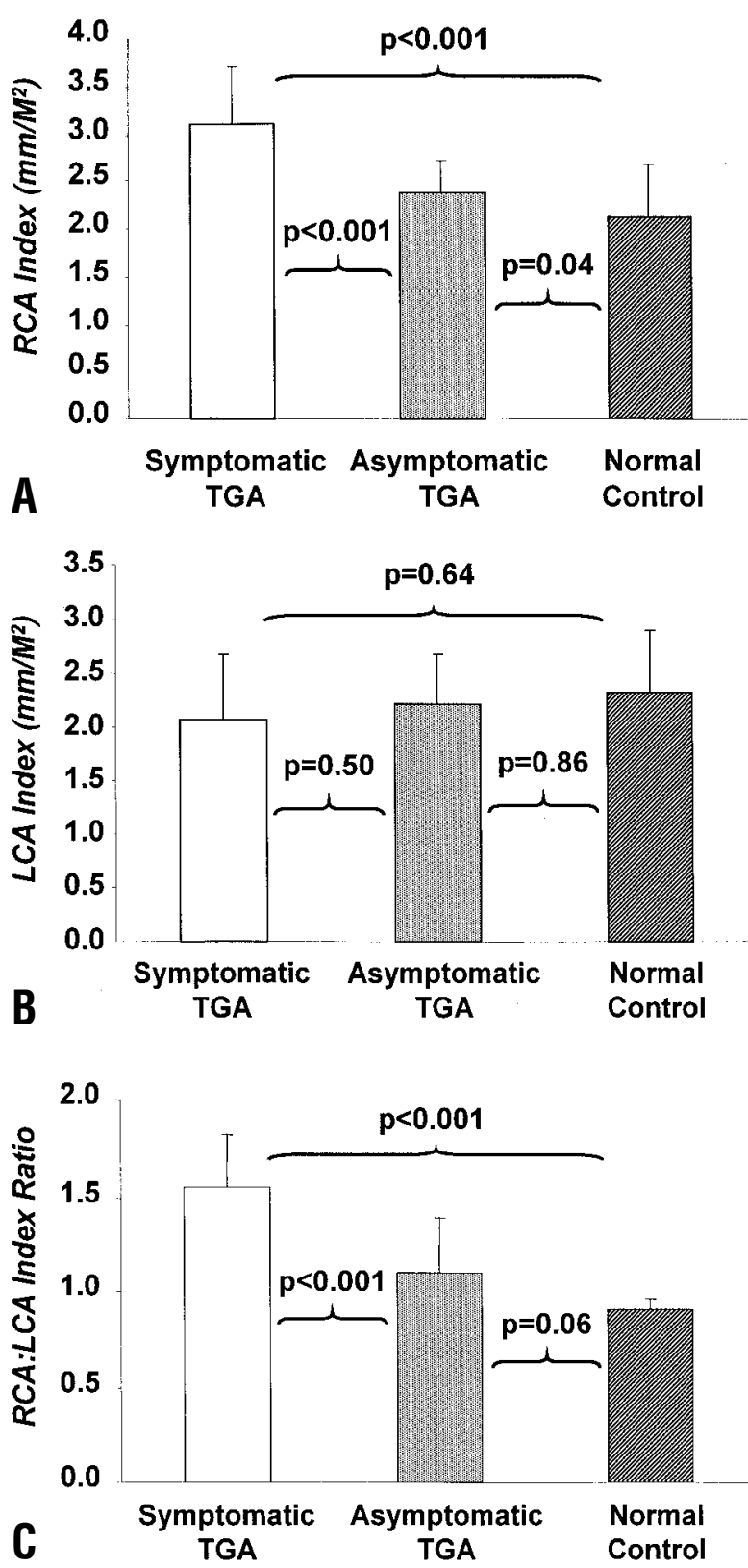

Fig 1. Bar graphs demonstrating (A) the angiographic diameter of the right coronary artery $(R C A)$ indexed to body surface area, (B) the angiographic diameter of the left coronary artery $(L C A)$ indexed to body surface area, and $(\mathbf{C})$ the ratio of indexed right and left coronary artery diameters in symptomatic and asymptomatic patients with TGA who have to undergo atrial inversion procedures, as well as in normal control patients. $P$ values are given for each 2-way comparison between groups, as determined by general factorial analysis of variance.

tricular failure and systemic atrioventricular valve regurgitation. Thus, these patients are frequently already sick. Before conversion to an arterial switch configuration in patients without left (pulmonary) ventricular outflow 


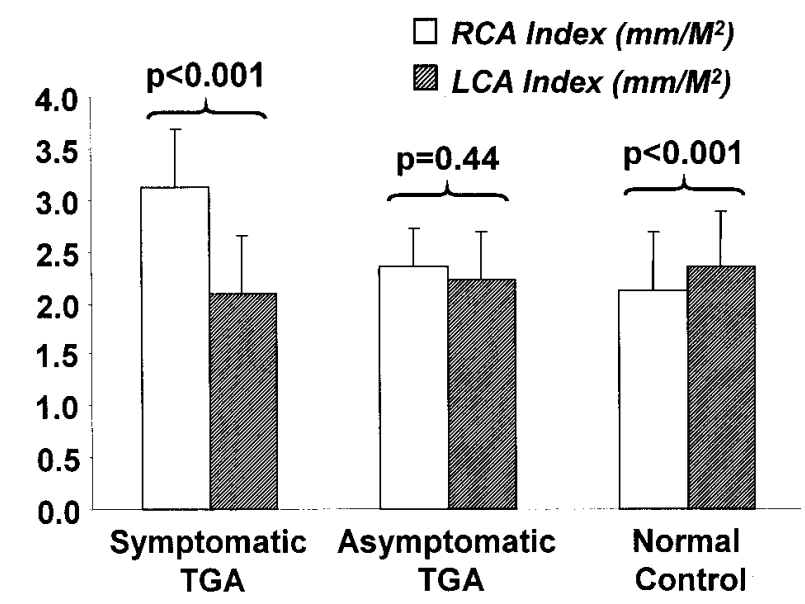

Fig 2. Bar graph demonstrating the relative magnitude of right $(R C A)$ and left $(L C A)$ coronary artery indices in patients with symptomatic TGA, patients with asymptomatic TGA, and normal control patients. $P$ values are given for comparisons of left and right coronary artery indices in each group.

obstruction, pulmonary artery banding is necessary. Banding is necessary because the wall thickness of the left ventricle is $60 \%$ to $80 \%$ of normal and the cavity diameter is $66 \%$ to $83 \%$ of normal as a result of the decreased afterload that the left ventricle faces in the pulmonary circulation. ${ }^{11,12}$ The purpose of pulmonary artery banding is to increase the afterload faced by the left ventricle in an effort to stimulate the acquisition of sufficient mass to serve the systemic circulation. It is not clear, however, whether this process of retraining imparts to the ventricle a hypertrophic response that will be sufficient to adapt to systemic afterload over the long term. Poor outcome in some patients is likely the result of inadequate acquisition of mass or otherwise ineffective adaptation to increased afterload.

Among the reasons that some patients with a failing atrial inversion procedure do not tolerate pulmonary artery banding and arterial switch may be decreased coronary flow reserve. Coronary flow reserve-in simple terms, the difference between autoregulated and maximally vasodilated coronary blood flow-is reduced in the hypertrophied ventricle. ${ }^{13-15}$ The causes of decreased coronary flow reserve include decreased myocardial capillary density, ${ }^{16}$ increased extravascular forces that may impede flow through the coronary vessels, ${ }^{17}$ coronary ostial narrowing, ${ }^{18}$ and the size of the large coronary arteries. ${ }^{19,20}$ In patients undergoing pulmonary artery banding and arterial switch, the size of the coronary arteries may be an important factor in limiting blood flow to the left ventricle. This is true especially when pulmonary artery banding is applied to increase left ventricular afterload and stimulate the acquisition of mass. Previous studies have shown that the coronary arterial response to ventricular hypertrophy is influenced by age of the patient, abruptness of the increased load, ventricular morphology (right vs left), and the presence or absence of ventricular failure. ${ }^{14,21}$

A small left coronary artery in patients who have undergone atrial level repair of TGA may contribute to decreased coronary flow reserve and subsequently inadequate retraining or chronic insufficiency of the left ventricle once it is forced to support the systemic circulation. It is known that the diameter of the coronary arteries is linearly related to the size and thickness of the ventricles ${ }^{7}$ and that in normal hearts the left coronary artery is larger than the right. Consistent with the relation between ventricular mass and coronary artery size, the left coronary artery is smaller in patients with unrepaired TGA than in patients without TGA. ${ }^{9}$ Interestingly, several reports have shown that the left coronary artery may remain smaller after the arterial switch operation.., 10

Increased left ventricular afterload after pulmonary artery banding increases left ventricular pressure and may restore the interventricular septum to a more normal configuration. However, although there is adequate hypertrophy after acutely increased left ventricular afterload by pulmonary artery banding in early infancy, when myocyte hyperplastic activity is still present, ${ }^{19,22-24}$ it is not clear whether microvascular coronary arterial density increases commensurately with myocyte hypertrophy in older patients. The ventricular hypertrophy that does occur may not be functionally adaptive. If microvascular coronary arterial density does not increase despite the acutely increased afterload, the larger coronary arteries may not be stimulated to dilate. In the face of increased left ventricular oxygen demand, patients may have a decrease in coronary flow reserve that prohibits the ventricle from supporting the increased afterload adequately. It is notable that left coronary artery size was smaller in patients with symptomatic TGA than in asymptomatic patients or control patients in our series.

Another interesting finding of the present study was that the right coronary artery in symptomatic patients was significantly larger than in patients with asymptomatic TGA or in control patients. As was found in the present series, the right coronary artery is larger than normal in patients who have had an atrial inversion procedure (even those without right ventricular failure), both in absolute terms and in relation to the size of the left coronary artery, because the right ventricle is thicker than the left ventricle. ${ }^{9}$ The even greater discrepancy in patients with ventricular dysfunction is likely a symptom of ventricular failure rather than a 
cause. Enlargement of the proximal right coronary artery may occur as the ventricle dilates in the face of atrioventricular valve regurgitation and/or pump failure, with the resulting increase in right ventricular oxygen demand. Another possible mechanism may include occult subaortic right ventricular outflow tract obstruction, which has been observed in patients with TGA, ${ }^{25}$ or possibly elevated pressure in the coronary sinus, which drained to the pulmonary venous pathway in all of our patients.

The interventricular septum plays an important role in the function of the right ventricle. Systemic pressure in the right ventricle makes the septum bulge toward the left ventricle. This pulls the chordae, which are attached to the septum, and causes tricuspid regurgitation. When pulmonary artery banding is performed in such patients, the septum is displaced toward the right ventricle and hence the tricuspid regurgitation improves. The anterior interventricular septum is supplied by the left anterior descending coronary artery, which is a branch of the left coronary artery. We speculate that the blood supply to the interventricular septum is compromised in patients who have a failing systemic ventricle. After pulmonary artery banding, the blood supply to the septum is further compromised, which may explain why some patients have undergone cardiac transplantation after banding. . $^{3,4}$

In conclusion, we have found that the right coronary artery is larger in patients with TGA than in normal control patients and larger in patients with a failing systemic right ventricle than in those with intact ventricular function. The left coronary artery also appears to be smaller in patients with ventricular failure after atrial inversion than in those without. These differences in the sizes of the proximal coronary arteries may be related to symptomatic status in patients with TGA who have undergone an atrial inversion procedure and may have implications for the efficacy of ventricular retraining. When pulmonary artery banding and subsequent arterial switch are considered for patients with a Mustard or Senning procedure and a failing systemic right ventricle, the size of the left coronary artery may be an important factor to consider and should be evaluated with preoperative imaging studies. Additional studies will be necessary to further explore this issue.

\section{Study limitations}

This study has some limitations. We were not able to obtain data on myocardial mass in all of the symptomatic patients, because of the retrospective nature of the study. A future prospective study will be able to address some of the questions that remain unanswered.
We express our gratitude to John D. Kugler, MD, for constructive criticism during revision of the manuscript.

\section{REFERENCES}

1. Wernovsky G, Mayer JE, Jonas RA, Hanley FL, Blackstone EH, Kirklin JW, et al. Factors influencing early and late outcome of the arterial switch operation for transposition of great arteries. $\mathrm{J}$ Thorac Cardiovasc Surg 1995;109:289-302.

2. Kirjavainen M, Happonen JM, Louhimo I. Late results of Senning operation. J Thorac Cardiovasc Surg 1999;117:488-95.

3. Mavroudis C, Backer CL. Arterial switch after failed atrial baffle procedures for transposition of the great arteries. Ann Thorac Surg 2000;69:851-7.

4. Chang AC, Wernovsky G, Wessel DL, Freed MD, Parness IA, Perry SB, et al. Surgical management of late right ventricular failure after Mustard or Senning repair. Circulation 1992;86(Suppl):II-140-9.

5. Cochrane AD, Karl TR, Mee RBB. Staged conversion to arterial switch for late failure of the systemic right ventricle. Ann Thorac Surg 1993;56:854-62.

6. Prieto LR, Latson LA, Flamm SD, Drumond-Webb J, Mee RBB. Conversion from atrial to arterial switch in patients with D-transposition of the great arteries [abstract]. Circulation 1998;97(Suppl):I-61.

7. O'Keefe JJ, Owen RM, Bove AA. Influence of left ventricular mass on coronary artery cross-sectional area. Am J Cardiol 1987;59:1395-7.

8. Smith A, Wilkinson JL, Arnold R, Dickinson D, Anderson RH. Growth and development of the ventricular walls in complete transposition of the great arteries (simple transposition). Am J Cardiol 1982;49:362-8.

9. Yatsunami K, Nakazawa M, Seguchi M, Momma K, Imai Y. The size of the coronary arteries in children with complete transposition before and after the arterial switch operation. Cardiol Young 1994:4:340-6.

10. Yatsunami K, Nakazawa M, Kondo C, Teshima H, Momma K, Takanashi Y, et al. Small left coronary arteries after arterial switch operation for complete transposition of great arteries. Ann Thorac Surg 1997;64:746-51.

11. Fogel MA, Gupta K, Baxter BC, Weinberg PM, Haselgrove J, Hoffman EA. Biomechanics of the deconditioned ventricle. Am J Physiol 1996;40:H1193-206.

12. Mahoney L, Turley K, Ebert P, Heymann MA. Long term results after atrial repair of transposition of the great arteries in early infancy. Circulation 1982;66:253-8.

13. Hoffman JIE. A critical view of the coronary reserve. Circulation 1987;75(Suppl):I-6-11.

14. O'Keefe DD, Hoffman JIE, Cheitlin R, O'Neil MJ, Allard JR, Shapkin E. Coronary blood flow in experimental canine left ventricular hypertrophy. Circ Res 1978;43:43-51.

15. Hoffman JIE. Maximal coronary flow and the concept of coronary vascular reserve. Circulation 1984;70:153-9.

16. Mueller TM, Marcus ML, Kerber RE, Young JA, Barnes RW, Abboudm M. Effects of renal hypertension and left ventricular hypertrophy on the coronary circulation in dogs. Circ Res 1978;42:543-9.

17. Harrison DG, Barnes DH, Hiratzka LF, Eastham CL, Kerber RE, Marcus ML. The effect of cardiac hypertrophy on the coronary collateral circulation. Circulation 1985;71:1135-45. 
18. Vogelberg K. Die Lichtungsweite der Coronarostien an Normalen und Hypertrophen Herzen. Z Kreislafforch 1957;46:101-6.

19. Di Donato RM, Fujii AM, Jonas RA, Castaneda AR. Age-dependent ventricular response to pressure overload. J Thorac Cardiovasc Surg 1992;104:713-22.

20. Linzbach AJ. Heart failure from the point of quantitative anatomy. Am J Cardiol 1960;5:370-82.

21. Bache RJ. Effects of hypertrophy on the coronary circulation. Prog Cardiovasc Dis 1988;31:403-40.

22. Boutin C, Wernovsky G, Sanders SP, Jonas RA, Castaneda AR, Colan SD. Rapid two stage arterial switch operation. Circulation 1994;90:1294-303.

23. Overy HR, Priest RE. Mitotic cell division in postnatal cardiac growth. Lab Invest 1966;15:1100-3.

24. Rudolph AM. Myocardial growth before and after birth: clinical implications. Acta Paediatr 2000;89:129-33.

25. Tomoharu A, Neirotti R, Becker AE. Is there an anatomic basis for subvalvular right ventricular outflow tract obstruction after an arterial switch repair for complete transposition? J Thorac Cardiovasc Surg 1993;105:142-6.

\section{Discussion}

Dr Roger B. B. Mee (Cleveland, Ohio). You have discussed some basic observations and measurements that are intriguing in terms of their significance and usefulness.

The big questions are these: Is this a chicken-and-egg discussion, and to what degree can the coronary arteries adapt to late imposed work demand? I suspect that at this stage you are still speculating.

If proximal arteries are unable to enlarge with added work demand, the late retraining of a thin-walled left ventricle would be a futile exercise. We know it can be done in some, but with some variation, and your article may hint at a subgroup in whom left ventricular retraining will prove more difficult to achieve.

If supply and demand remain reasonably linked, then late retraining is feasible, but clearly should be done gradually. That is something we have learned merely from observing ventricular function after pulmonary artery banding, particularly in older patients.

The small left coronary artery has been suggested as a primary cause of right ventricular dysfunction in the symptomatic Mustard/Senning group. This is, I believe, your correct hypothesis. However, I would like some extra information. I think proximal coronary artery size should be correlated with right and left ventricular indexed mass, so as to sort out the issue of supply and demand.

I have several questions. First, in comparing left coronary artery sizes in symptomatic and asymptomatic Mustard/ Senning groups, was there any difference in left ventricular pressure? You said there was no left ventricular obstruction, but to clinch your thesis, I think you have to know the left ventricular pressure and mass. For instance, if the left ventricular mass in the symptomatic patients with the smallest left coronary arteries is greater than or equal to the left ventricular mass in the asymptomatic patients, then I think you have a very good case. Do you have this information?

Second, are you aware of any other situation in the body where healthy supply arteries are unable to respond gradually to increased end-organ vascular demand? What comes to light in my mind are the change in size of a radial artery when a shunt is inserted for long-term hemodialysis and the change in size in supply arteries going to a vascular tumor. If we had evidence anywhere else that this supply-and-demand rule was not met in the mature patient, then that would also reinforce your hypothesis.

The final question is this: Is the right coronary artery larger in symptomatic Mustard/Senning patients simply because the right ventricular mass is larger? If the right ventricle is dilated, usually it has larger mass. I think the next step is to closely evaluate indexed ventricular mass, and that is probably best done by magnetic resonance imaging.

Dr Amin. Thank you. I will try to answer your last question first.

I will try to use an analogy. If you create a fistula between the coronary artery and a low-pressure right ventricle, the coronary artery size will increase in time. Therefore, I think we do have evidence that the coronary artery size will increase. The reason it does not increase in patients with TGA, I believe, is that the mitotic activity at the cellular level is lost in the first year of life. Once the pulmonary artery banding is done, flow to the ventricle will not increase because there is no cellular growth to stimulate more flow through the coronary artery. This is the same situation encountered with a fistula. I think the answer to your question is, yes, the artery can grow, whether it be a coronary artery or any other artery.

I do not have exact information about ventricular mass, but we did compare ventricular mass between asymptomatic and symptomatic patients when adequate imaging was available. We did not find any difference between them. Unfortunately, we did not have adequate data in all of the patients regarding myocardial mass. The pressures in the left ventricle were normal. I do not have any mean or median data, but pressures were normal, and there was no statistically significant difference between these 2 groups.

\section{Timely}

The Journal of Thoracic and Cardiovascular Surgery delivers the information you need now. Articles usually appear within four months of acceptance. 